\title{
The lipid metabolism gene FTO influences breast cancer cell energy metabolism via the PI3K/AKT signaling pathway
}

\author{
YAZHUO LIU ${ }^{1}$, RUOYU WANG $^{2}$, LICHUAN ZHANG $^{3}$, JIANHUA LI $^{4}, \mathrm{KELI} \mathrm{LOU}^{5}$ and BINGYIN SHI ${ }^{6}$ \\ Departments of ${ }^{1}$ Metabolic Diseases and Nutritional Disorders, ${ }^{2}$ Oncology, ${ }^{3}$ Respiratory Medicine, ${ }^{4}$ Nursing, and \\ ${ }^{5}$ Metabolic Diseases and Nutritional Disorders, Affiliated Zhongshan Hospital of Dalian University, Dalian, Liaoning 116001; \\ ${ }^{6}$ Department of Endocrinology, The First Affiliated Hospital of Xi'an Jiaotong University, Xi'an, Shaanxi 710061, P.R. China
}

Received July 21, 2016; Accepted February 22, 2017

DOI: $10.3892 / \mathrm{ol} .2017 .6038$

\begin{abstract}
The present study assessed the effect of the lipid metabolism, fat mass and the obesity-associated gene (FTO), on energy metabolism of breast cancer cells. The human breast cancer cell lines, MCF-7 and MDA-MB-231, and HCC1937 human breast cells were studied. Real-time PCR was used to measure the levels of FTO mRNA from breast cancer cells and normal breast cells. MDA-MB-231 cells were transfected with miFTO inhibitor or inhibitor control, and cells were assessed for levels of lactic acid, ATP, pyruvate kinase activity, and hexokinase activity assay using specific kits. Western blot analysis was used to measure the levels of phosphatidylinositol 3-kinase (PI3K), p-PI3K, protein kinase B (Akt) and p-Akt in transfected breast cancer cells. The expression of FTO was significantly increased in MCF-7 and MDA-MB-231 cells compared with HCC1937 cells $(\mathrm{P}<0.01)$. The lactic acid content of breast cancer cells transfected with the miFTO inhibitor was significantly lower compared with cells transfected with the miFTO inhibitor control and nontransfected cells $(\mathrm{P}<0.05)$. The ATP content of breast cancer cells transfected with the miFTO inhibitor was significantly lower compared with the control group and inhibitor control group $(\mathrm{P}<0.05)$. The pyruvate kinase activity and hexokinase activity of breast cancer cells transfected with the miFTO inhibitor were significantly lower compared with the control group and inhibitor control group $(\mathrm{P}<0.01)$. Western blot analysis showed that after breast cancer cells were transfected with the miFTO inhibitor, the levels of PI3K, p-PI3K, Akt and p-Akt were significantly lower than in the control group and inhibitor control group. In conclusion, the FTO gene is overexpressed in breast cancer cells. Overexpression of the FTO gene can promote breast
\end{abstract}

Correspondence to: Dr Bingyin Shi, Department of Endocrinology, The First Affiliated Hospital of Xi'an Jiaotong University, 277 Yanta West Street, Xi'an, Shaanxi 710061, P.R. China

E-mail: shi_by1@163.com

Key words: breast cancer, energy metabolism, fat mass and obesity-associated, phosphatidylinositol 3-kinase/protein kinase B cancer cell glycolysis and the mechanism is related to the PI3K/AKT signaling pathway.

\section{Introduction}

Breast cancer is a common malignant tumor in women. According to statistics, breast cancer accounts for $10 \%$ of malignant tumors and its incidence is second to that of uterine endometrial carcinoma (1). There are several causes of breast cancer. Early detection is difficult, women aged 40-60 years are at high risk for breast cancer, and its incidence is highest during the peri-menopausal period (2). Since breast cancer relapses and metastasizes easily, and has poor prognosis, there are great challenges in diagnosis and treatment of breast cancer.

In recent years, obesity was shown to increase the risk of a variety of diseases (3). Additional studies revealed that obesity related genes, such as fat mass and obesity-associated (FTO) are widely expressed in the human body (4). The FTO gene was found to be overexpressed in prostate cancer, pancreatic cancer, endometrial cancer and liver cancer. Its overexpression affected the energy metabolism of cancer cells, and was closely related to the occurrence and development of cancer (5). To our knowledge, there are no studies on the effect of FTO gene expression on breast cancer cell energy metabolism. In the present study, we used breast cancer cells as a model to explore the relationship between FTO gene expression and energy metabolism, and performed preliminary studies on its mechanism of action, to provide a new potential target for the treatment and diagnosis of breast cancer.

\section{Materials and methods}

Cells. The human breast cancer cell lines (MCF-7 and MDA-MB-231), and human breast cells (HCC1937) purchased from Cell Bank of Chinese Academy of Sciences (Shanghai, China) were used. Additional instruments and reagents used are shown in Table I. MCF-7, MDA-MB-231 and HCC1937 cells were removed from storage in liquid nitrogen and thawed in a water bath set to $37^{\circ} \mathrm{C}$. Cells were then added to culture medium [Dulbecco's modified Eagle's medium/F12 (DMEM/F12) supplemented with $5 \%$ fetal bovine serum (FBS), $0.1 \mathrm{mg} / \mathrm{ml}$ streptomycin, $100 \mathrm{U} / \mathrm{ml}$ penicillin and $2 \mathrm{mmol} / 1$ glutamine]. Cells were grown in culture bottles in an incubator $\left(37^{\circ} \mathrm{C}\right.$, 
$5 \% \mathrm{CO}_{2}$ ) for $48 \mathrm{~h}$. Culture medium was removed when cells reached $90 \%$ confluence. Cells were trypsinized in $0.25 \%$ trypsin, and centrifuged at $800 \mathrm{x} \mathrm{g}$ for $10 \mathrm{~min}$ at room temperature. Cells were washed with DMEM/F12 and then seeded again in cell culture bottles.

RT-PCR. TRIzol was added to cell lysis buffer for lysis of MCF-7, MDA-MB-231 and HCC1937 cells that were in the logarithmic growth phase. After 5 min of digestion, lysates were placed in new Eppendorf (EP) tubes (Corning, Inc., Corning, NY, USA), and $200 \mu 1$ chloroform was added. Solutions were shaken up and down 15 times, and placed at room temperature for $5 \mathrm{~min}$. Samples were then centrifuged $\left(8,500 \mathrm{x} \mathrm{g}, 4^{\circ} \mathrm{C}, 10 \mathrm{~min}\right)$. RNA in the supernatant was transferred to new EP tubes. Next, $75 \%$ ethanol was added and samples were centrifuged $\left(6,500 \mathrm{x} \mathrm{g}, 4^{\circ} \mathrm{C}, 5 \mathrm{~min}\right)$. The supernatant was discarded, and solutions were placed on a super-clean worktable to air dry. DEPC water (Biosharp, Hefei, China) was then added and mixed well. The concentration and purity of RNA were determined by UV spectrophotometry. According to the instructions of the reverse transcription kit, RNA was reverse transcribed into cDNA. Real-time PCR amplification of cDNA was performed to measure the expression of FTO mRNA in each group of cells.

Transfection. After trypsinization of MDA-MB-231 cells in the logarithmic growth phase, cells were washed with DMEM/ F12, cell growth medium was added, and the cell concentration was adjusted to $2 \times 10^{5} / \mathrm{ml}$. Cells were then seeded in 6-well culture plates, and placed at $37^{\circ} \mathrm{C}, 5 \% \mathrm{CO}_{2}$ for $48 \mathrm{~h}$. When cells were $50 \%$ confluent, growth medium was replaced with incomplete culture medium (without FBS), and placed at $37^{\circ} \mathrm{C}$, $5 \% \mathrm{CO}_{2}$ for $1 \mathrm{~h}$. The incomplete, serum-free medium was mixed with miFTO inhibitor or inhibitor control, and incubated at $37^{\circ} \mathrm{C}$ for 5 min (solution A). The serum-free medium was mixed with Lipofectamine 2000 (solution B) (KeyGen, Nanjing, China). Next, solutions A and B were mixed, and left to incubate at room temperature for $20 \mathrm{~min}$. The cell culture medium was discarded, and cells were repeatedly washed with phosphate-buffered saline (PBS). The transfection reagent and miRNA were added to the cell culture plates, and cells were placed at $37^{\circ} \mathrm{C}, 5 \% \mathrm{CO}_{2}$ for $6 \mathrm{~h}$. The medium was replaced with complete culture medium, and cells were left in the incubator for an additional $48 \mathrm{~h}$.

Measurement of lactic acid content in culture medium of breast cancer cells after transfection. MDA-MB-231 cells were transfected with miFTO inhibitor, or inhibitor control and left to incubate at $37^{\circ} \mathrm{C}, 5 \% \mathrm{CO}_{2}$ for $48 \mathrm{~h}$. Culture supernatant was harvested and transferred to EP tubes, and centrifuged ( $800 \mathrm{x} \mathrm{g}, 5 \mathrm{~min})$. The content of lactic acid in supernatant was measured according to the instructions of the Sigma Lactic Acid Test kit (Sigma-Aldrich, St. Louis, MO, USA).

Detection of ATP content in transfected cells. Transfected MDA-MB-231 cells grown in the incubator $\left(37^{\circ} \mathrm{C}, 5 \% \mathrm{CO}_{2}\right)$ for $48 \mathrm{~h}$ were trypsinized and washed with PBS. Cell culture medium was added to resuspend the cells, then cells were transferred to EP tubes and centrifuged ( $800 \mathrm{x}$ g, $5 \mathrm{~min})$. The supernatant was discarded. Cells were washed twice with
PBS and the supernatant was discarded after centrifugation at $800 \mathrm{x}$ g for $3 \mathrm{~min}$. The cells were mixed homogeneously with ultrapure water. The cell homogenates were transferred to EP tubes and heated in a water bath $\left(100^{\circ} \mathrm{C}, 10 \mathrm{~min}\right)$. The content of ATP was determined according to the instructions of the ATP test kit.

Detection of hexokinase and pyruvate kinase activity in breast cancer cells. MDA-MB-231 cells were transfected with miFTO inhibitor or inhibitor control for $48 \mathrm{~h}$. The activity of hexokinase and pyruvate kinase were detected according to the instructions of the hexokinase and pyruvate kinase test kits.

Western blot analysis. After $48 \mathrm{~h}$ culture, culture medium from transfected MDA-MB-231 cells was discarded, cells were washed with PBS and lysed on ice for $30 \mathrm{~min}$. Protein extracts were mixed with loading buffer, and boiled at $100^{\circ} \mathrm{C}$ for $5 \mathrm{~min}$. A total volume of $50 \mu \mathrm{l}$ of the denatured protein samples were loaded on gels (12\% separation gel and 5\% spacer gel). A voltage of $80 \mathrm{~V}$ was applied to samples and then adjusted to $120 \mathrm{~V}$ when protein reached the separation gel. When bromophenol blue entered the separation gel, electrophoresis was stopped. Protein was transferred to PVDF membranes overnight at $4^{\circ} \mathrm{C}$. PVDF membranes were washed with TBST 3 times, and blocked with skim milk for $2 \mathrm{~h}$ at $37^{\circ} \mathrm{C}$. Membranes were treated with primary antibody overnight at $4^{\circ} \mathrm{C}$, washed with TBST, and incubated with secondary antibody for $1.5 \mathrm{~h}$ at room temperature. Membranes were washed and developed. Protein expression was analyzed with the Odyssey scanning system (LI-COR, Inc., Lincoln, NE, USA). Primary rabbit polyclonal AKT antibody (dilution, 1:500; cat. no. ab38449); rabbit monoclonal p-AKT antibody (dilution, 1:500; cat. no. ab81283), rabbit monoclonal PI3K antibody (dilution, 1:500; cat. no. ab86714), rabbit polyclonalp-PI3K antibody (dilution, 1:500; cat. no. ab182651) and secondary goat antirabbit (HRP) IgG antibody (dilution, 1:2,000; cat. no. ab6721) were all purchased from Abcam (Cambridge, MA, USA).

Statistical analysis. Data were analyzed with SPSS 16.0 statistical software (SPSS, Inc., Chicago, IL, USA). Comparisons between groups were by t-test. $\mathrm{P}<0.05$ was considered to indicate a statistically significant difference.

\section{Results}

Measurement of FTO mRNA expression in breast cancer cells and breast cells by RT-PCR. In the logarithmic growth phase, MCF-7, MDA-MB-231 and HCC1937 cells were harvested for extraction of total RNA. The expression levels of FTO in the 3 groups of cells were detected by real-time quantitative PCR. The relative expression levels of FTO mRNA in MCF-7 cells was $26.89 \pm 2.31,36.23 \pm 2.91$ in MDA-MB-231 cells and $8.96 \pm 3.01$ in HCC1937 cells. The levels of FTO mRNA in MCF-7 and MDA-MB-231 cells were significantly higher than in $\mathrm{HCC} 1937$ cells $(\mathrm{P}<0.01)$ (Fig. 1).

Measurement of lactic acid and ATP content. The content of lactic acid and ATP in MDA-MB-231 cells transfected with the miFTO inhibitor or inhibitor control were detected according to the lactic acid test kit and ATP test kit. After $48 \mathrm{~h}$ 
Table I. Major instruments and reagents.

Instruments and reagents

Sources

Enzyme-labeled instrument

Ultraviolet spectrophotometer

$\mathrm{CO}_{2}$ incubator

Laminar flow cabinet

Inverted microscope

PCR instrument

Centrifuge

RevertAid First Strand cDNA Synthesis kit

DMEM/F12 culture medium

Lactic acid test kit

ATP content test kit

Pyruvate kinase test kit

Hexokinase test kit

Real-time fluorescent quantitative PCR kit

Agarose

Antibody dilution

FBS

Protein concentration test kit

Mycillin

Trypsin

RNA isolating reagent kit

Cell total protein extraction kit

PBS

P13K monoclonal antibody

p-P13K monoclonal antibody

AKT monoclonal antibody

p-AKT monoclonal antibody

HRP-anti-antibody
Nanjing Detie Laboratory Equipment Co., Ltd., Nanjing, China

Thermo Fisher Scientific, Inc., Waltham, MA, USA

Sanyo, Tokyo, Japan

Suzhou Purification Equipment Co., Ltd., Suzhou, China

Nikon, Tokyo, Japan

Beckman Coulter, Inc., Brea, CA, USA

Hunan Hengnuo Instrument Equipment Co., Ltd., Changsha, China

Beyotime Institute of Biotechnology, Haimen, China

Sigma-Aldrich, St. Louis, MO, USA

Sigma-Aldrich, St. Louis, MO, USA

Sigma-Aldrich, St. Louis, MO, USA

Sigma-Aldrich, St. Louis, MO, USA

Sigma-Aldrich, St. Louis, MO, USA

Thermo Fisher Scientific, Inc., Waltham, MA, USA

Thermo Fisher Scientific, Inc., Waltham, MA, USA

MultiSciences (Lianke) Biotech Co., Ltd., Hangzhou, China

Hangzhou Sijiqing Biology Engineering Materials Co., Ltd., Hangzhou, China

Beyotime Institute of Biotechnology, Hangzhou, China

Sigma-Aldrich, St. Louis, MO, USA

Sigma-Aldrich, St. Louis, MO, USA

Beyotime Institute of Biotechnology, Haimen, China

Beyotime Institute of Biotechnology, Haimen, China

SinoBio Biotech Co., Ltd., Shanghai, China

Abcam, Cambridge, MA, USA

Abcam, Cambridge, MA, USA

Abcam, Cambridge, MA, USA

Abcam, Cambridge, MA, USA

Abcam, Cambridge, MA, USA

DMEM, Dulbecco's modified Eagle's medium; FBS, fetal bovine serum; PBS, phosphate-buffered saline; P13K, phosphatidylinositol 3-kinase; AKT, protein kinase B.

culture, the lactic acid content of the miFTO inhibitor group was $8.97 \pm 0.25 \mathrm{mmol} / \mathrm{l}$, the lactic acid content of the inhibitor control group was $17.11 \pm 1.02 \mathrm{mmol} / \mathrm{l}$, and the lactic acid content of the blank control group was $17.08 \pm 1.32 \mathrm{mmol} / 1$. The lactic acid content of breast cancer cells transfected with miFTO inhibitor was significantly lower compared with the control group and inhibitor control group. FTO mRNA inhibitors can inhibit the production of lactic acid in breast cancer cells (Fig. 2A). After 48 h culture, the ATP content of the miFTO inhibitor group was $31.45 \pm 1.58 \mathrm{mmol} / \mathrm{l}$, the ATP content of the inhibitor control group was $44.12 \pm 3.12 \mathrm{mmol} / 1$, and the ATP content of the blank control group was $44.56 \pm 2.45 \mathrm{mmol} / \mathrm{l}$. The ATP content of breast cancer cells transfected with the miFTO inhibitor was significantly lower compared with the control group and inhibitor control group $(\mathrm{P}<0.05)$. FTO mRNA inhibitors can inhibit the production of ATP in breast cancer cells (Fig. 2B).

Detection of pyruvate kinase and hexokinase activity. The activity of hexokinase and pyruvate kinase in MDA-MB-231

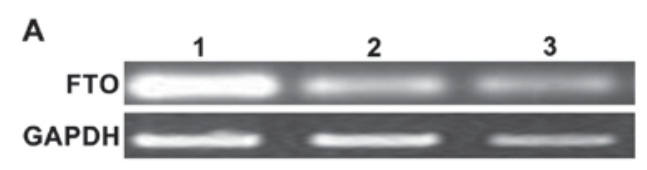

B

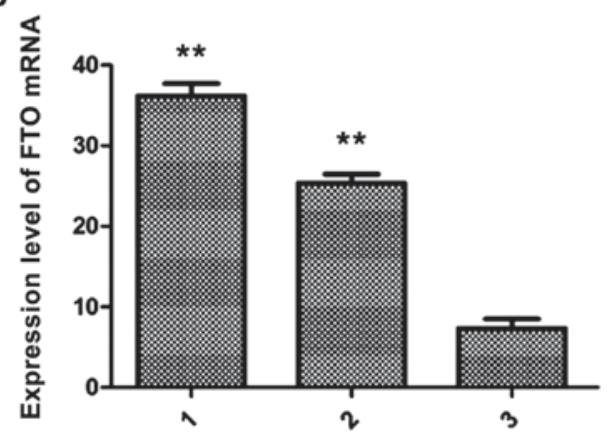

Figure 1. Relative FTO mRNA expression levels in MCF-7, MDA-MB-231 and HCC1937 cells by RT-PCR. (A) FTO mRNA expression level detected by agarose gel electrophoresis. (B) FTO mRNA expression level. Lane 1, MDA-MB-231; lane 2, MCF-7; and lane 3, HCC1937; ${ }^{* *} \mathrm{P}<0.01$ vs. HCC1937. FTO, fat mass and obesity-associated. 
A

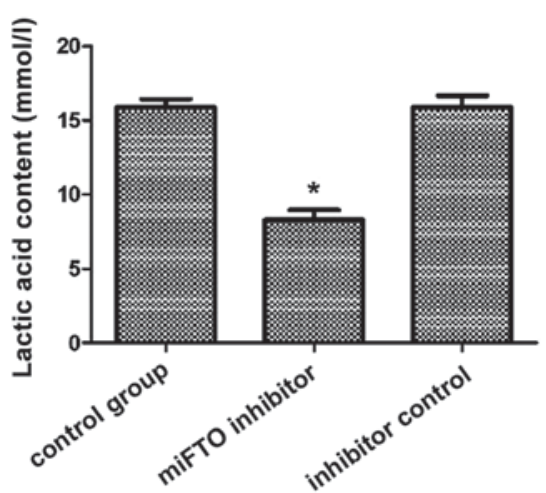

B

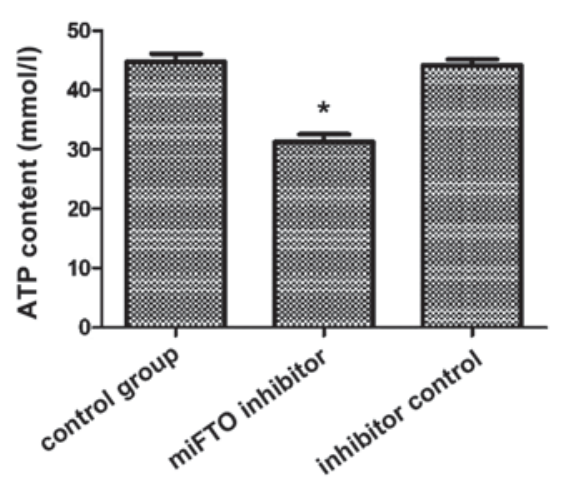

Figure 2. Lactic acid content and ATP content after transfection of MDA-MB-231 cells. (A) Lactic acid content and (B) ATP content; ${ }^{*} \mathrm{P}<0.05$ vs. control group.

A

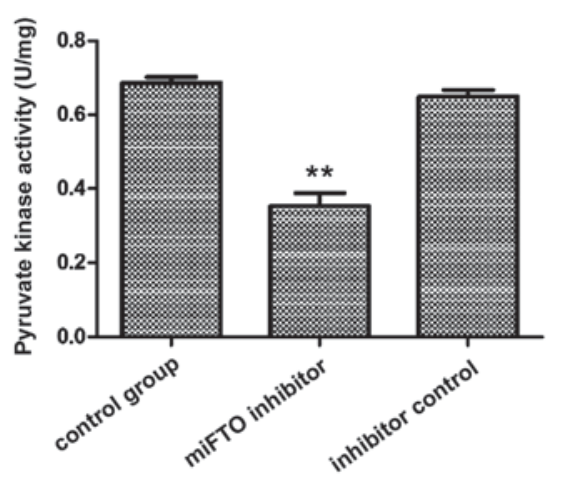

B

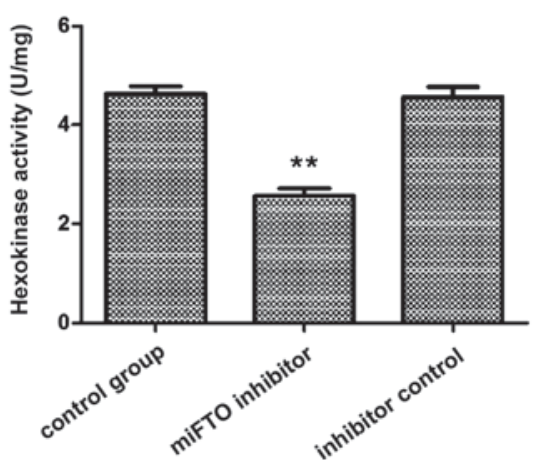

Figure 3. Pyruvate kinase and hexokinase activity in breast cancer cells. (A) Pyruvate kinase activity and (B) hexokinase activity; ${ }^{* *} \mathrm{P}<0.01$ vs. control group.

A
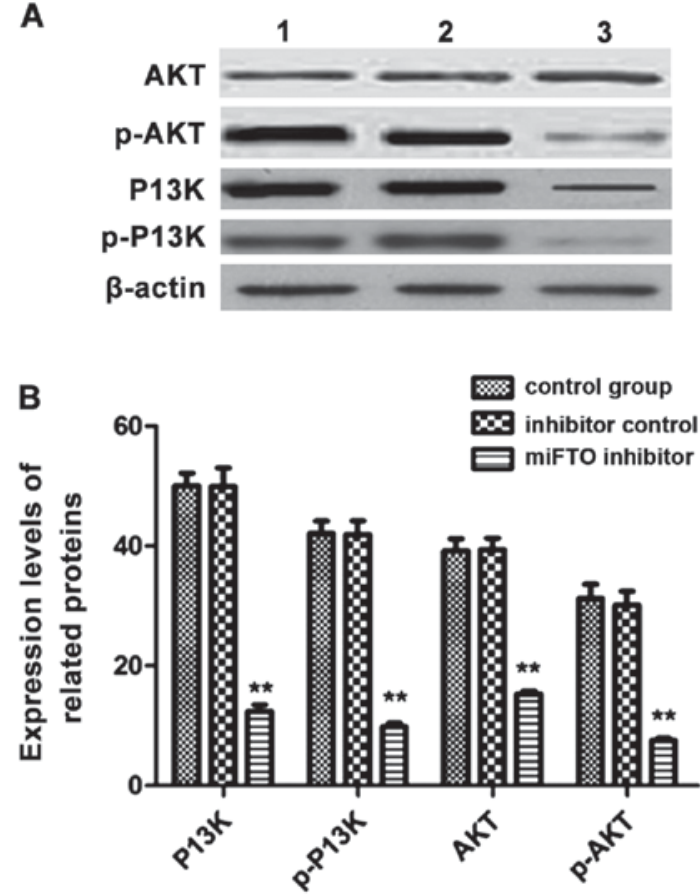

Figure 4. The expression levels of PI3K, p-PI3K, AKT and p-AKT in breast cancer cells by western blot analysis. (A) The expression levels of PI3K/AKT signaling proteins by western blot analysis. (B) The relative protein expression levels. Lane 1, control group; lane 2, inhibitor control group; and lane 3 , miFTO inhibitor group; ** $\mathrm{P}<0.01$ vs. control group. PI3K, phosphatidylinositol 3-kinase; AKT, protein kinase B. cells transfected with miFTO inhibitor or inhibitor control were detected according to the instructions of the hexokinase and pyruvate kinase test kits. The results showed that pyruvate kinase activity in breast cancer cells transfected with miFTO inhibitor and inhibitor control were $0.39 \pm 0.01$ and $0.68 \pm 0.02$, respectively, and $0.71 \pm 0.03$ in the nontransfected cells. Pyruvate kinase activity of breast cancer cells transfected with miFTO inhibitor was significantly lower compared with the control group and inhibitor control group $(\mathrm{P}<0.01)$. Hexokinase activity in breast cancer cells transfected with the miFTO inhibitor and inhibitor control were $2.54 \pm 0.21$ and $4.86 \pm 0.25$, respectively, and $4.84 \pm 0.20$ in the nontransfected cells. Hexokinase activity of breast cancer cells transfected with the miFTO inhibitor was significantly lower compared with the control group and inhibitor control group $(\mathrm{P}<0.01)$. Therefore, miFTO inhibitors can reduce the activity of hexokinase and pyruvate kinase in breast cancer cells (Fig. 3).

Western blot analysis to detect the expression levels of related proteins in cells. MDA-MB-231 cells transfected with the miFTO inhibitor or inhibitor control for $48 \mathrm{~h}$ were harvested, and lysates were used to analyze the expressions of phosphatidylinositol 3-kinase (PI3K), p-PI3K, protein kinase B (Akt), and $\mathrm{p}-\mathrm{AKT}$ by western blot analysis. After transfection with the miFTO inhibitor, the expressions of PI3K, p-PI3K, AKT and p-AKT were significantly lower compared with the control 
group and inhibitor control group. The phosphorylated forms of PI3K and AKT decreased significantly (Fig. 4).

\section{Discussion}

Breast cancer is a common malignant tumor in women. According to statistics, the incidence of breast cancer accounts for $10 \%$ of all malignant tumors. There are 1.3 million newly diagnosed cases of breast cancer worldwide every year, and $\sim 50$ million people die from breast cancer each year. Therefore, breast cancer is a serious threat to the health of women. The occurrence of breast cancer has regional differences. The morbidity of breast cancer in developed countries is higher than that in developing countries (6). There are several causes of breast cancer and it is difficult to detect in the early stage. Research on the pathogenesis of breast cancer is important for treatment and diagnosis.

In recent years, studies have shown that obesity can increase the risk of breast cancer. Obese women were 3 times more likely to have breast cancer than nonobese women (3). The lipid metabolism gene FTO, has been found to be closely related to obesity. The FTO gene contains nine exons and is located on chromosome 16 . It is widely expressed in adults and in the fetus. FTO is most highly expressed in the pituitary, pancreatic islets, hypothalamus and adrenal glands $(7,8)$. FTO is overexpressed in prostate cancer, pancreatic cancer, hepatocellular carcinoma and endometrial carcinoma. It therefore has a close relationship with the occurrence and development of cancer. In this study, we used human breast cancer cells (MCF-7 and MDA-MB-231) and human breast cells (HCC1937) to determine the levels of FTO mRNA by real-time fluorescence quantitative PCR. The results showed that the FTO mRNA levels in breast cancer cells were significantly higher than in normal breast cells, suggesting that FTO is an oncogene, which represents a potential new marker for the early diagnosis of breast cancer.

Cell energy metabolism is the process of transforming organic matter into energy. In normal cells, ATP is produced by oxidative decomposition of glucose, which can be divided into aerobic oxidation and glycolysis $(9,10)$. Glucose can be oxidized to produce ATP under aerobic conditions, while under anoxic conditions, ATP can be generated by glycolysis (11). The energy metabolism of tumor cells is different from that of normal cells. In cases where tumor cells receive sufficient oxygen, energy is also produced by glycolysis which converts pyruvate to lactic acid (the 'Warburg effect') (12). Studies have shown that aerobic glycolysis can be found in lung cancer, breast cancer, colon cancer and renal cancer cells (13). Pyruvate kinase and hexokinase play a key role in glycolysis. In tumor cells, hexokinase exists as isozymes. The expression of hexokinase is related to the occurrence and development of colon cancer and renal cell carcinoma. Downregulated expression of pyruvate kinase can inhibit the production of lactic acid by glycolysis (14). In this study, after cells were transfected with the FTO mRNA inhibitor, the ATP levels in breast cancer cells decreased, pyruvate kinase and hexokinase activity decreased significantly, and the content of lactic acid in the medium decreased significantly. These results demonstrate that overexpression of the FTO gene could promote glycolysis in breast cancer cells.
The role of the PI3K/AKT signaling pathway in tumor cells is an area of intense study $(15,16)$. The PI3K/AKT signaling pathway is related to the proliferation and apoptosis of cancer cells, and can regulate the activity of caspase-9 (17), p53 (18), Bad (19), and other proteins, and inhibit apoptosis. The PI3K/AKT signaling pathway is active in several types of cells, and it plays an important role when cells are under hypoxic conditions. Under hypoxic conditions, the PI3K/AKT signaling pathway can upregulate insulin, epidermal growth factor and cytokine expression. It can deliver messages to protein tyrosine kinases via transmembrane receptors, activate PI3K, and catalyze the generation of PIP3, which then delivers messages to Akt, and activates the Ras-MAPK signaling pathway, which consequently causes a series of complex reactions in the body $(20,21)$. In this study, through transcriptional inhibition of the FTO gene, the protein expression of $\mathrm{PI} 3 \mathrm{~K}, \mathrm{p}-\mathrm{PI} 3 \mathrm{~K}, \mathrm{Akt}$ and p-Akt in cells increased significantly according to western blot analysis, demonstrating that expression of the FTO gene affected the energy metabolism of breast cancer cells through the PI3K/AKT signaling pathway.

In conclusion, overexpression of FTO in breast cancer cells can result in upregulation of pyruvate kinase and hexokinase activity, increase the amount of ATP generation in cells, and promote glycolysis and lactic acid production. FTO overexpression affects the energy metabolism of breast cancer cells, and the mechanism is related to the PI3K/AKT signaling pathway. Our results represent a potential new therapeutic option for the treatment and diagnosis of breast cancer.

\section{References}

1. Piccart-Gebhart MJ, Procter M, Leyland-Jones B, Goldhirsch A, Untch M, Smith I, Gianni L, Baselga J, Bell R, Jackisch C, et al; Herceptin Adjuvant (HERA) Trial Study Team: Trastuzumab after adjuvant chemotherapy in HER2-positive breast cancer. $\mathrm{N}$ Engl J Med 353: 1659-1672, 2005.

2. Dowsett M, Forbes JF, Bradley R, Ingle J, Aihara T, Bliss J, Boccardo F, Coates A, Coombes RC, Cuzick J, et al; Early Breast Cancer Trialists' Collaborative Group (EBCTCG): Aromatase inhibitors versus tamoxifen in early breast cancer: patient-level meta-analysis of the randomised trials. Lancet 386: 1341-1352, 2015.

3. Gallagher EJ and LeRoith D: Obesity and diabetes: the increased risk of cancer and cancer-related mortality. Physiol Rev 95: 727-748, 2015.

4. Sevgi M, Rigoux L, Kühn AB, Mauer J, Schilbach L, Hess ME, Gruendler TO, Ullsperger M, Stephan KE, Brüning JC, et al: An obesity-predisposing variant of the FTO gene regulates D2R-dependent reward learning. J Neurosci 35: 12584-12592, 2015.

5. Milagro FI, Moreno-Aliaga MJ and Martinez JA: FTO obesity variant and adipocyte browning in humans. N Engl J Med 374: 190-191, 2016.

6. Kuchenbaecker KB, Ramus SJ, Tyrer J, Lee A, Shen HC, Beesley J, Lawrenson K, McGuffog L, Healey S, Lee JM, et al; EMBRACE; GEMO Study Collaborators; Breast Cancer Family Registry; HEBON; KConFab Investigators; Australian Cancer Study (Ovarian Cancer Investigators); Australian Ovarian Cancer Study Group; Consortium of Investigators of Modifiers of BRCA1 and BRCA2: Identification of six new susceptibility loci for invasive epithelial ovarian cancer. Nat Genet 47: 164-171, 2015.

7. Hasstedt SJ, Coon H, Xin Y, Adams TD and Hunt SC: $A P O H$ interacts with $F T O$ to predispose to healthy thinness. Hum Genet 135: 201-207, 2016.

8. Salgado-Montilla J, Rodríguez-Caban J, Gonzalez-Sepulveda L, Sanchez-Ortiz R and Irizarry-Ramirez M: Presence of FTO rs9939609 and rs9930506 and severity of prostate cancer in Puerto Ricans. Cancer Res (106th Annual Meeting Abstracts): 4836, 2015. 
9. Barbier-Torres L, Delgado TC, García-Rodríguez JL, ZubieteFranco I, Fernández-Ramos D, Buqué X, Cano A, Gutiérrez-de Juan V, Fernández-Domínguez I, Lopitz-Otsoa F, et al: Stabilization of LKB1 and Akt by neddylation regulates energy metabolism in liver cancer. Oncotarget 6: 2509-2523, 2015.

10. Roberts DJ and Miyamoto S: Hexokinase II integrates energy metabolism and cellular protection: akting on mitochondria and TORCing to autophagy. Cell Death Differ 22: 248-257, 2015.

11. Giménez-Cassina A and Danial NN: Regulation of mitochondrial nutrient and energy metabolism by BCL-2 family proteins. Trends Endocrinol Metab 26: 165-175, 2015.

12. Valenti D, Vacca RA and de Bari L: 3-Bromopyruvate induces rapid human prostate cancer cell death by affecting cell energy metabolism, GSH pool and the glyoxalase system. J Bioenerg Biomembr 47: 493-506, 2015.

13. Gatenby RA and Gillies RJ: Why do cancers have highaerobic glycolysis? Nat Rev Cancer 4: 891-899, 2004.

14. Vincent EE, Sergushichev A, Griss T, Gingras MC, Samborska B, Ntimbane T, Coelho PP, Blagih J, Raissi TC, Choinière L, et al: Mitochondrial phosphoenolpyruvate carboxykinase regulates metabolic adaptation and enables glucose-independent tumor growth. Mol Cell 60: 195-207, 2015.

15. Liang M, Liu J, Ji H, Chen M, Zhao Y, Li S, Zhang X and Li J: A Aconitum coreanum polysaccharide fraction induces apoptosis of hepatocellular carcinoma (HCC) cells via pituitary tumor transforming gene 1 (PTTG1)-mediated suppression of the P13K/Akt and activation of p38 MAPK signaling pathway and displays antitumor activity in vivo. Tumour Biol 36: 7085-7091, 2015.
16. Zhang CZ, Wang XD, Wang HW, Cai Y and Chao LQ: Sorafenib inhibits liver cancer growth by decreasing mTOR, AKT, and PI3K expression. J BUON 20: 218-222, 2015.

17. Zhu JJ, Cui Y, Cui K, Li X and Zhang ZY: Distinct roles of parafibromin in the extracellular environment, cytoplasm and nucleus of osteosarcoma cells. Am J Transl Res 8: 2426-2431, 2016.

18. Mock CD, Jordan BC and Selvam C: Recent advances of curcumin and its analogues in breast cancer prevention and treatment. RSC Advances 5: 75575-75588, 2015.

19. Yang Z, Xie C, Xu W, Liu G, Cao X, Li W, Chen J, Zhu Y, Luo S, Luo Z, et al: Phosphorylation and inactivation of PTEN at residues Ser380/Thr382/383 induced by Helicobacter pylori promotes gastric epithelial cell survival through PI3K/Akt pathway. Oncotarget 6: 31916-31926, 2015.

20. Pande M, Bondy ML, Do KA, Sahin AA, Ying J, Mills GB, Thompson PA and Brewster AM: Association between germline single nucleotide polymorphisms in the PI3K-AKT-mTOR pathway, obesity, and breast cancer disease-free survival. Breast Cancer Res Treat 147: 381-387, 2014.

21. Bains M and Roberts JL: Estrogen protects against dopamine neuron toxicity in primary mesencephalic cultures through an indirect P13K/Akt mediated astrocyte pathway. Neurosci Lett 610: 79-85, 2016 